\title{
IMPACT Database of Traumatic Brain Injury: Design and Description
}

\author{
ANTHONY MARMAROU, ${ }^{1}$ JUAN LU, ${ }^{1}$ ISABELLA BUTCHER, ${ }^{2}$ \\ GILLIAN S. MCHUGH, ${ }^{2}$ NINO A. MUSHKUDIANI, ${ }^{3}$ GORDON D. MURRAY, ${ }^{2}$ \\ EWOUT W. STEYERBERG, ${ }^{3}$ and ANDREW I.R. MAAS ${ }^{4}$
}

\begin{abstract}
The objective of this report is to describe the design and content of the International Mission for Prognosis And Clinical Trial (IMPACT) database of traumatic brain injury which contains the complete dataset from most clinical trials and organized epidemiologic studies conducted over the past 20 years. This effort, funded by the U.S. National Institutes of Health, has led to the accumulation thus far of data from 9205 patients with severe and moderate brain injuries from eight randomized placebo controlled trials and three observational studies. Data relevant to the design and analysis of pragmatic Phase III clinical trials, including pre-hospital, admission, and post-resuscitation assessments, information on the acute management, and short- and long-term outcome were merged into a top priority data set (TPDS). The major emphasis during the first phase of study is on information from time of injury to post-resuscitation and outcome at 6 months thereby providing a unique resource for prognostic analysis and for studies aimed at optimizing the design and analysis of Phase III trials in traumatic brain injury.
\end{abstract}

Key words: coma; databank; head injury; IMPACT; traumatic brain injury

\section{INTRODUCTION}

$\mathbf{T}$ The ATTEMPTS by NIH-supported studies and industry to improve outcome from severe brain injury by pharmacologic means and tested in clinical trials have led to many disappointments. Several investigators have reasoned that in many instances the pre-clinical studies were lacking in providing a clear demonstration of drug penetration into the brain or were inadequate in testing the formulation on a variety of injury models (Narayan et al., 2002). In addition, a clear demonstration of mechanism of action of the drug was not evident from experimental and clinical studies. Others argued that positive experimental studies in rodents are simply not translatable to human head injury and have considered cell replacement therapy as an alternative (Schouten et al., 2004). However, few questioned if the drug effect was simply overwhelmed by the heterogeneity of the injury or that a

IMPACT: International Mission on Prognosis and Analysis of Clinical Trials in TBI.

${ }^{1}$ Department of Neurosurgery, Virginia Commonwealth University Medical School, Richmond, Virginia.

${ }^{2}$ Public Health Sciences, University of Edinburgh Medical School, Edinburgh, United Kingdom.

${ }^{3}$ Center for Medical Decision Making, Department of Public Health, and ${ }^{4}$ Department of Neurosurgery, Erasmus Medical Center, Rotterdam, The Netherlands. 


\section{MARMAROU ET AL.}

positive effect of the drug was not detected using the outcome scales currently available. The issues of center effects, variations in management, lack of surrogate markers, broad inclusion criteria, and insensitive outcome scales all remain a significant problem. Many of these issues can be addressed and their possible solution provided the raison $d$ ẹtre for compilation of head injury data by many groups (Beretta et al., 2003; Braakman et al., 1980; Citerio et al., 2000; Fearnside et al., 1993; Marshall, 1988; Murray et al., 1999a). However, despite the arduous collection of data, most studies were observational and did not take advantage of the quality control inherent in placebo controlled head injury clinical trials sponsored by industry and the NIH. As a result, we perceived a need to develop a major resource which could be used to develop and assess new approaches to the design and analysis of Phase III trials in traumatic brain injury (TBI) (Maas et al., 2006). We proposed to the NIH that data from available randomized controlled trials (RCTs) and observational studies in TBI conducted from 1984 to the present would be combined. We estimated that this would yield a database of some 10,000 patients from which we could test hypotheses relating to diagnostic criteria, management, and novel statistical methods for clinical trial design and analysis. Our objective was to bring together in one master database the wealth of information that was collected during the implementation of major head injury trials conducted during the past 20 years. This has been accomplished, and the IMPACT (International Mission for Prognosis And Clinical Trial Design) database contains at present information from 9205 head-injured patients. The objective of this report is to describe the methods and procedures by which the IMPACT database came into realization.

\section{METHODS}

The project was organized as a collaborative venture between the Erasmus University in Rotterdam, The Netherlands, the University of Edinburgh, Scotland, and the Virginia Commonwealth University Medical College in Richmond, Virginia. The objective was to include as many major Phase III trials and epidemiological studies as was feasible. The project is on-going and we are continuing to negotiate access to additional studies. Gaining permission from original investigators and, where relevant, commercial sponsors, is a complex exercise. Fortunately, this was facilitated by the IMPACT investigators at Richmond, Rotterdam, and Edinburgh having senior roles in the American Brain Injury Consortium (ABIC) (Marmarou, 1996) and the European Brain Injury Consortium (EBIC) (Teasdale et al., 1997). Indeed, it is part of the requirements for $\mathrm{ABIC}$ and $\mathrm{EBIC}$ when taking on a TBI study that the data should ultimately be made available for use in academic projects such as IMPACT.

The first 11 studies to be incorporated into the IMPACT database are specified in Table 1. These comprise eight randomized controlled trials and three epidemiological studies. Specific details of each of these studies are given later.

\section{Sequence of Events}

Each IMPACT center was responsible for extracting the variables from the individual source data in their possession. It is widely recognized that industry-sponsored clinical trials tend to have record forms which capture far more data than are useful in practice. A key early phase of the IMPACT project was to document carefully exactly what data were available for each study, and to prioritize the extraction of a key set of variables. This was driven by our objective of building a resource which could be used to develop and test new methodologies, but we also had to take account of the pragmatic issue that we needed to choose variables which were each recorded for a significant number of the constituent studies.

This data extraction was guided by a data dictionary and original study documentation which standardized the format of variables entered into the top priority data set. The overall process was enormously labor intensive, and required considerable clinical insight. Timeframes were particularly difficult to standardize over studies. Epidemiological studies tend to take the time of injury as "time zero" and relate the time of subsequent observations or events back to this reference time. In contrast, clinical trials tend to take the time of randomization as "time zero" and relate other times back to this time. Further, some clinical trials take "days" as successive 24-h periods starting at the time of randomization, when others take the first day as the day of randomization, and start "day 2" at midnight on the day of randomization. Appropriate conventions needed to be developed and agreed to make time-related data as consistent as possible over all the constituent studies.

Another major issue was establishing a consistent set of categories for coding each variable. For example, the presence of hypoxia on admission was coded as "no" or "yes" for some studies, and coded as "no," "suspect," or "definite" for others. Variables such as cause of injury raised this same issue but in a far more complex form. Our guiding principle was never to discard information, and so the most expansive codings are recorded on the database. In addition, numerous "derived" variables collapse these original codings to ones more suited for analy- 


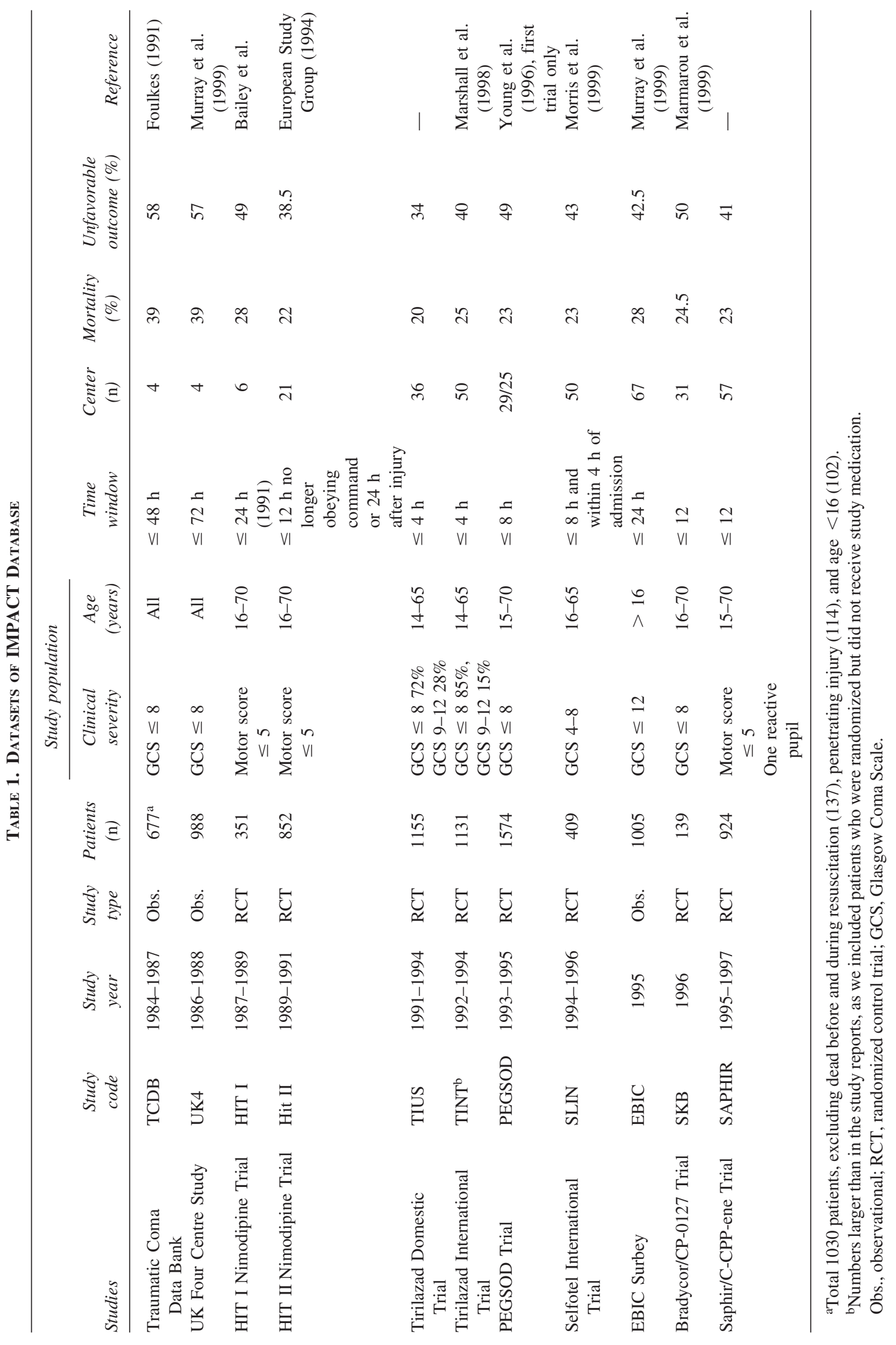




\section{MARMAROU ET AL.}

sis across datasets. So, for example, suspect and definite for hypoxia would be collapsed to "yes," to have a consistent no/yes coding over all of the individual studies.

For most studies, the extracted data were compared with results from published papers, as a check of the accuracy of the extraction process. Once the initial data extraction was complete, data sets were transferred to Richmond, and a merged IMPACT SAS database was created and circulated amongst the three centers. As the work progressed a number of problems with the interpretation of codings and with the original study documentation came to light. These have been resolved on an on-going basis, and updates of the database circulated.

\section{RESULTS}

\section{Basic Design Features of IMPACT}

The patients comprising the IMPACT database are head injured patients accrued either into an observational study or entered into a RCT. All data had been collected prospectively. In the case of an RCT, the patient randomization into placebo or study treatment was known. A severe head injury was defined as having a Glasgow Coma Scale (GCS) score (Teasdale and Jennett, 1974) of 8 or less. Patients with a GCS score of 9 to 12 were considered moderately injured. In two trials, HIT I and HIT II, the head injury was classified on the basis of motor score 1-5. Pre-hospital information concerns data that were recorded prior to admission to the study hospital and included both pre-hospital information obtained from emergency services and information from referring (first) hospitals. Penetrating injuries such as gunshot wounds or patients not surviving transport to a study hospital were excluded. Taking these limits into consideration, the age of patients in the IMPACT database ranged from 1 to 93 years, and the average age equaled 34 years (SD 16 years). The overall gender distribution was $77 \%$ males and $23 \%$ females.

\section{Description of Clinical Databases Merged into IMPACT}

A summary of the eight randomized controlled trials and three observational studies targeted for merging into IMPACT and covering a data collection period of 1984-1997 is shown in Table 1. Taken in combination, they sum to 9205 patients. Most injuries were severe $(82 \%)$ as defined by a derived GCS $\leq 8$ or a motor score $\leq 5$, and only three trials included moderate brain injury. Of the 9205 patients, 6535 were included within randomized clinical trials and 2670 from observational studies. A brief description of each database is included in the following section to illustrate the diversity of data within the studies.

\section{TCDB (Traumatic Coma Data Bank): An Observational Study}

The TCDB (1984-1987) was the first observational study of severe brain injury sponsored by the National Institutes of Health (NIH) and totaled 1030 patients collected by four centers in the United States located in Charlottesville (Virginia), Richmond (Virginia), Galveston (Texas), and San Diego (California) (Foulkes et al., 1991). All patients presented at these centers with a GCS $\leq 8$ within $48 \mathrm{~h}$ after injury were included in the TCDB database. We excluded patients dying prior to hospital admission $(n=137)$, patients with penetrating injury $(n=114)$, and patients less than 16 years of age $(n=102)$. A total of 677 patients were included as the portion of TCDB merged into IMPACT. The pre-hospital, first-hospital, and admission hospital information was available. In transit observations were considered as prehospital. Admission to one of the four TCDB study hospitals was considered as the study hospital "admission" data. Although the computed tomography (CT) classification system was developed by the TCDB, the TCDB data only contain the CT classification at discharge. The Glasgow Outcome Scale score (GOS) was assessed at 6 months post-injury.

\section{UK4 (The UK Four Centre Study): An Observational Study}

The UK4 Centre Study (1986-1988) was an observational study containing 988 patient records collected by four centers in the United Kingdom: Edinburgh, Glasgow, Liverpool, and Southampton. Patients were enrolled if they were admitted to one of the centers within 3 days of severe head injury, defined as resulting in coma (no eye opening, no comprehensible response, and not obeying commands). Data were available in a single electronic file. The data were crosschecked against tables in a published paper (Murray et al., 1999b) and found to be in agreement with the downloaded electronic version. Recordings at pre-hospital and post stabilization time points were not available. No blood pressure readings or pre-enrollment lab values were recorded. For pre-enrollment secondary insults, presence/absence was indicated for hypotension and hypoxia only. The dataset included a variable indicating whether or not a CT scan was performed; however, only the presence of epidural, subdural or intracerebral hematomas was recorded. Thus, CT classification, cisterns, shift, and traumatic subarachnoid hemorrhage (tSAH) could not be scored. No clear definition of the size of lesions was available and distinction 
between single and multiple contusions was not possible. Outcome according to the GOS was assessed at 3 and 6 months for 976 patients (12 missing) and 984 patients (four missing), respectively, although the date of outcome was not available. The outcome scale used in UK4 includes three additional categories to the standard fivenamely, $6=$ out of hospital, $7=$ vegetative state or severe disability indistinguishable at 1 month, and $8=$ moderate disability/good recovery.

\section{HIT I: An RCT Evaluating the Effect of Nimodipine}

HIT I (1987-1989) was the first multi-center study investigating the efficacy of Nimodipine in patients with severe head injury (Bailey et al., 1991). This study was conducted in six centers from the United Kingdom and Finland, and enrolled 351 subjects. Patients were enrolled into this study if they were not obeying commands and randomization could be performed within $24 \mathrm{~h}$ after injury. The dataset of HIT I consisted of 25 data files and one comments file. Paper copies of all the forms were maintained by the Edinburgh study group. The data were originally stored as character files in column format with no text headings or identifiers in the files. Each dataset had a corresponding descriptive file which specified the column names, formats, and position in the dataset. The extraction of the data into a readable format was very time consuming. Of the 25 data files able to be extracted, only one read in accordance with the descriptive file specified. The remaining data files could be viewed but not read into any statistical package. The paper forms had to be compared with the dataset in order to decipher the placement, format and names of columns for all 25 data files. The extracted data were checked against the published paper and found to agree. Subjects were assessed initially, monitored twice daily for up to 7 days and then assessed at 6 months after injury according to the GOS. The Marshall CT classification was not available although the presence of hematomas and contusions was recorded by the investigator. The study publication reports an absolute difference in outcome of $4 \%$ in favor of Nimodipine-treated patients.

\section{HIT II: The Second RCT Evaluating the Effect of Nimodopine}

HIT II (1989-1991) was the second multi-center randomized trial of Nimodipine in TBI, conducted in 21 centers from 13 European countries. A total of 852 patients were randomized into this trial (European Study Group on Nimodipine in Severe Head Injury, 1994). Patients were eligible if they could not obey commands at the time of assessment for entry into the trial and had undergone CT.
The stipulated time window for this study was possibility for initiation of treatment with the test drug within $24 \mathrm{~h}$ after injury and within $12 \mathrm{~h}$ of the patient not obeying commands. The case report form (CRF) for the study was available, along with documentation for 17 data files mostly corresponding with sections on the CRF. However, of these data files, only 10 were available, including one for the evaluation of CT (not shown on the CRF). Files not available included data on adverse events, medication, accompanying diseases, laboratory values, and follow-up outcome assessment. The data extracted for IMPACT were crosschecked against tables in a published paper and found to be in agreement. Pre-enrollment hypotension and hypothermia were determined using cut-off points of systolic $\mathrm{BP} \leq 90 \mathrm{~mm} \mathrm{Hg} /$ body temperature $\leq 35^{\circ} \mathrm{C}$, respectively, at any point up to and including entry to trial. Pre-enrolment hypoxic events could not be determined.

Variables representing first hospital and post-stabilization mean arterial blood pressure, respectively, were calculated using the average systolic/diastolic values available at these time points. In practice, these average values were the only ones available for the vast majority of cases. The CT classification (Marshall Scale) was only available from the evaluation of the CT file, and not by investigator. Outcome GOS at 6 months was recorded for 819 patients (33 missing), along with a corresponding date. The study publication reported an absolute difference of $1 \%$ in favor of Nimodipine-treated patients. In a subgroup of patients with tSAH the difference was $8 \%$.

\section{TINT and TIUS (Tirilazad Trials): An RCT Evaluating the Effect of Tirilazad}

The international Tirilazad trial (TINT; 1992-1994) and the North American Tirilazad trial (TIUS; 19911994) were conducted simultaneously and investigated the efficacy of the drug Tirilazad Mesylate, an inhibitor of lipid peroxidation in TBI. TINT enrolled 1131 patients in 50 centers from 15 countries (Marshall et al., 1998). TIUS included data on 1155 patients enrolled in the United States and Canada (36 centers). Both trials enrolled patients aged 15-65 years, with a severe (GCS 3-8) or moderate (GCS 9-12) closed TBI. In accordance with the protocol, the proportion of patients with moderate TBI was lower in the international trial than in the North American Trial (14\% vs. 28\%). The time window for study drug administration for this study was $4 \mathrm{~h}$. The data files for the Tirilazad trials were extremely complex comprising over $500 \mathrm{MB}$ spread over 109 files for TIUS and 94 for TINT. Additionally, there were 175 files with specific instructions for the codification and storage of the files. These files however primarily contained particular instructions for creation of the data and did not describe or document the contents of the files. Data extraction was 


\section{MARMAROU ET AL.}

further complicated by a lack of correspondence in some variables between the coding in the database and the coding in the case record forms. Due to the complexity of the data and unclear documentation in some key variables, extensive data checking through both frequency and graphic approaches were applied. The data extracted for TINT were crosschecked against tables in the published paper and found to be in general agreement. The TINT and TIUS data were more complete than all other studies in the IMPACT database and included pre-hospital, admission and postresuscitation neurological evaluations, admission CT classification, secondary insults, and hourly monitoring. Both 3- and 6-month outcomes were available. Results of TIUS have not been published. A $2 \%$ difference in outcome exists in favor of the placebo population. The study publication on TINT reports a $3 \%$ difference in outcome in favor of the placebo population. Post-hoc subgroup analysis of males with tSAH showed a significantly lower mortality in patients on active treatment, but no difference in favorable outcome. TIUS was stopped just before completion because of concerns by the monitoring committee about an excess number of deaths in patients treated with Tirilazad, but on full analysis there was no significant difference in the distribution of the GOS.

\section{SLIN: An RCT of the NMDA Antagonist Selfotel}

The effect of the competitive NMDA receptor antagonist Selfotel (CGS 197555) has been studied in two parallel studies, one (the international arm) in Canada, Western Europe, Australia, and Argentina, (SLIN) and the other in the United States and Israel. Results of these combined studies have been published (Morris et al., 1999). We had access to an extract of the data from the international arm of the Selfotel study. This arm included data from 409 patients with severe TBI enrolled in 50 centers. Patients were included with severe head injury $(\mathrm{GCS} \leq 8)$ aiming at treatment initiation within $8 \mathrm{~h}$ of injury and within $4 \mathrm{~h}$ of admission. The SLIN trial includes mostly post-resuscitation neurological evaluation, admission CT diagnoses, and hourly monitoring data. Both 3- and 6-month outcomes were available. The studies were halted prematurely, because of concerns of the Safety and Monitoring Committee about an increased number of deaths and severe brain-related adverse events occurring in the drug-treated groups in two contemporaneously conducted trials in stroke patients. Although analysis of the data from the head-injured patients did not show an excess of adverse events in the Selfotel-treated group, further futility analysis indicated a low likelihood of demonstrating a major benefit on pursuing the trial to completion.

\section{PEGSOD: An RCT of Pegulated SuperOxide Dismutase (Peg-SOD)— A Free Radical Scavenger}

The PEGSOD trial (1993-1995) comprised two randomized placebo controlled trials, both conducted in the United States, investigating the efficacy of pegulated super-oxide dismutase, a free radical scavenger, in traumatic brain injury. The total sample size equaled 1,562 from trials designated $05(n=463)$ and $06(n=1099)$. The results from trial 05 have been published (Young et al., 1996). Of these patients, 651 were assigned to placebo, and 633 patients to $10,000 \mathrm{U}$, and 278 patients to $20,000 \mathrm{U}$ of Peg-SOD. The trial, 05 conducted in 29 centers and 06 conducted in 25 centers, enrolled patients with a severe TBI (GCS $\leq 8)$ with a time window of 8 h. Pre-hospital, first hospital, and admission to study hospital information, demographic information, blood gas, laboratory information, and 3-month outcomes were extracted from the raw dataset. The studies did not have admission CT classification and hourly information. The extracted admission CTs were classified as either normal CT, or presence of subdural, epidural, intraventricular hemorrhage, and cerebral contusions. In general, the PEGSOD study included complete early endpoints (14-day mortality and GOS, discharge mortality and GOS), and strong laboratory data, such as liver functions, blood gas, and whole blood counts. In the PEGSOD raw datasets, there were 12 patients identified in addition to the 1562 reported patients. The data extraction was unable to separate these patients from the main dataset, and therefore these records were also included in the current IMPACT database. Outcome in the PEGSOD trial was only assessed at 3 months after injury. On combined analysis of the PEGSOD trials, a $4 \%$ shift towards favorable outcome in patients on active treatment has been reported (Muizelaar et al., 1995; Muizelaar and Miller, 2001).

\section{EBIC: An Observational Study by the European Brain Injury Consortium}

The EBIC core data (1995) contains one file with records on 1005 subjects from 67 centers throughout Europe (Murray et al., 1999a). Subjects were assessed initially, at discharge, and at 6 months after injury. Subjects with a severe (GCS $\leq 8$ ) or a moderate head injury (GCS 9-12) recorded at any GCS assessment (pre-hospital, at the Accident \& Emergency Department, post-resuscitation, or admission to the neurosurgical unit) were included in the study. Paper copies of all of the forms are maintained by Edinburgh. An annotated CRF was available for the EBIC data, making the process of extracting information relatively straightforward. The data have been published, and the raw and extracted data have been 


\section{THE IMPACT DATABASE OF TBI}

checked against the published paper. All variables were checked and any inconsistencies noted. Initial data recorded include GCS, time of injury and admission, basic demographics, secondary insults, CT classification, based on the Marshall scoring system and information on subarachnoid haemorrhage. Data recorded at discharge include the worst CT classification, intracranial and systemic complications, disability rating scale and dates of discharge and death. The CT scans were assessed by the local investigator at each center.

Of the 67 centers which responded to the survey, 55 provided 6-month outcome data. These outcome data were $94 \%$ complete with 796 out of 847 possible responses. The remaining 12 centers were not able to provide any data on 6-month outcome on any patient. Therefore, in total approximately $17 \%$ of the total 1005 subjects have a missing outcome measure although for two distinct reasons. GOS was only measured once at 6 months and not at any earlier time points.

\section{SKB: The RCT of a Bradykinin Antagonist- Bradycor}

The SKB trial was a Phase II prospective randomized clinical trial of Bradycor, a bradykinin antagonist, which was conducted in 1996 at 31 centers within North America (Marmarou et al., 1999). Patients with a GCS of 3-8 with at least one reactive pupil were randomized with a treatment window of $12 \mathrm{~h}$ after injury. The trial was halted after recruitment of 139 patients because of results of animal toxicology studies conducted during the course of the trial. The information relevant to the IMPACT project was extracted from the datasets dated January 99, but outcomes and CTs were not available. The outcomes and CT information (central reader) were obtained from the ABIC database. The SKB combined with the ABIC data set contained a relatively complete CT diagnoses, hourly monitoring data and both 3- and 6-month outcomes. The study publication reports a $12 \%$ improvement in favorable outcome in patients on active treatment.

\section{SAPHIR: An RCT of a Novel NMDA Antagonist and Placebo}

The Saphir study (SAP) was a Phase III trial evaluating the efficacy of the competitive NMDA antagonist DCPP-ene and was conducted during 1995-1997 in 57 European centers. Inclusion criteria consisted of patients not obeying commands with at least one reactive pupil. Initiation of treatment was within $12 \mathrm{~h}$ of injury. The study enrolled 924 patients. Despite the fact that the study was initiated over 10 years ago, the full study results have not yet been published. Paper copies of the data forms were not available. The data were originally stored in zipped
SAS files with embedded formats. The data have been unzipped and formatted in SAS. The data files were very complex in format, comprising some $265 \mathrm{MB}$ of data spread over 41 files each with a unique data structure, making the selection of the correct variables and records difficult. A partially annotated CRF was available. However, the annotations in many cases did not match with the data files. The investigator assessment of the CT was only scored as normal or abnormal and a Marshall classification of CT was only available on central review. The data were checked against an unpublished Novartis clinical study report and found to be in reasonable agreement. The Saphir dataset included both 3- and 6-month outcome assessed according to the GOS. No indication of study drug effects have been reported to investigators.

\section{Strategy for Combining the Diverse Clinical Trial Data: The Top Priority Data Set}

Our strategy for developing the IMPACT database was to subdivide the dataset and initially consider those parameters describing the clinical events from moment of injury to post-stabilization. This was referred to as the Top Priority Data Set (TPDS). The information entered into the TPDS from injury to first hospital admission is shown in Table $2 \mathrm{a}$. The selected variables from first hospital to study hospital are shown in Table 2b. In brief, we (a) provided a compilation of the source data (complete data set) in electronic format; (b) extracted the TPDS variables from the source data into a preliminary select database; and (c) transmitted the select database to Richmond. At Richmond, all the selected databases were reformatted according to the data dictionary described earlier which defined the structure of each variable. A second round of checks on the merged TPDS resolved errors in merging and re-coding. This process resulted in a TPDS, which contains the information necessary for prognostic analysis and to test our hypotheses and complete our objectives.

\section{Pooling Placebo and Treatment Data}

The fundamental question of pooling placebo and treated patients was addressed. As part of the process of negotiating access to the constituent datasets, we wished to assure the original investigators that there was no intention to "data dredge" and reanalyze the datasets in an attempt to find evidence of treatment effects which may have been missed in the original analyses. As no trial showed a significant difference between placebo and drug, it was felt that pooling the data would be appropriate for almost all of the analyses which are planned to be undertaken. The IMPACT database does however contain the randomization codes for the clinical trial databases, and 


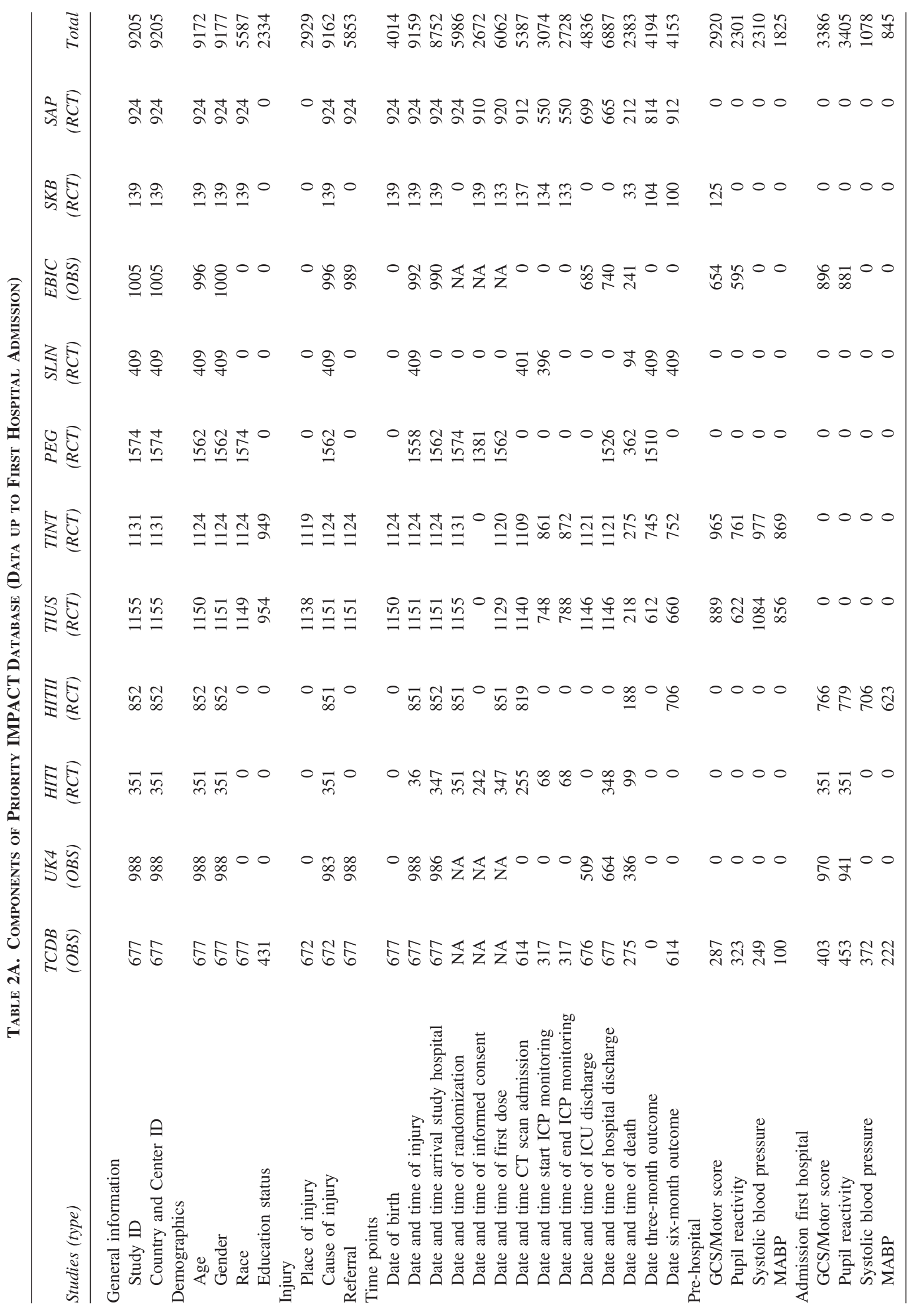




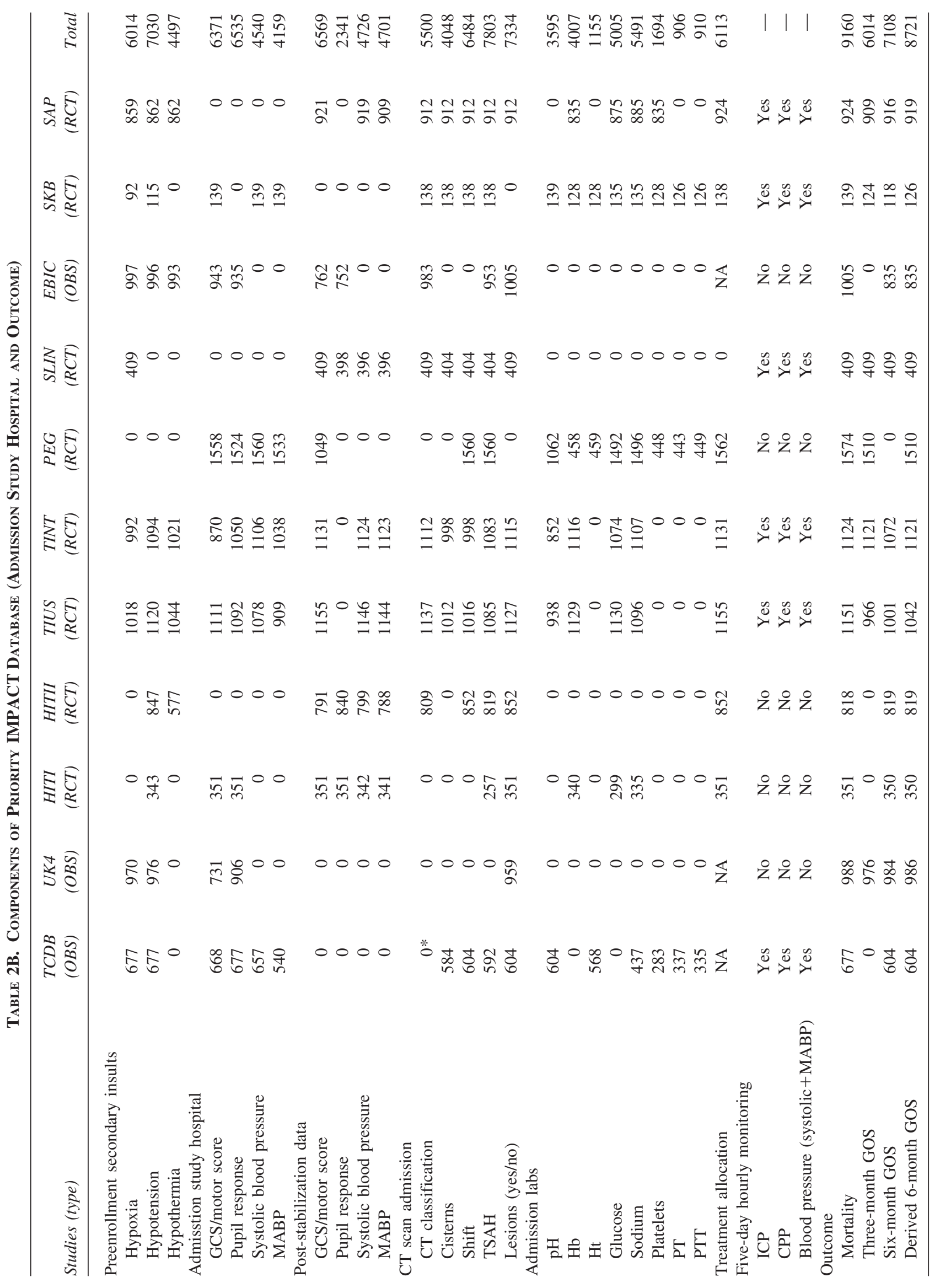




\section{MARMAROU ET AL.}

so it will be possible to restrict an analysis to, say, placebo patients only, where this is thought to be important.

\section{Derived Glasgow Coma Scale Score}

In the various studies included in the IMPACT database, the GCS prior to study enrollment was scored at different time periods. Some datasets only included the randomization GCS, others further details on the GCS pre-hospital, on admission to referring hospital during transit or on admission to study hospital. These variable time points for assessment of the GCS illustrate the complexity involved in reformatting the datasets into a common structure for further analysis. For purposes of a univariate analysis and prognostic modeling where an accurate GCS was required, a derived GCS was defined as the GCS closest to randomization or in the case of the observational studies, the GCS upon entry into the study. The GCS post-stabilization was the first selected and if this was not available, then the GCS on admission to the study hospital would be selected. In this way, the patient numbers available for prognostic studies were maximized. The problem of missing values or untestable scores is common among databases and in the case of IMPACT, it was decided to merge missing/untestable cases into one category for the GCS components.

\section{Derived Glasgow Outcome Scale Score}

Wherever possible, 6-month GOS was extracted (Jennett and Bond, 1975). A further derived variable was created where, for patients without 6-month GOS recorded, the 3-month GOS was imputed if available. This was required in particular for the PEGSOD study (Muizelaar et al., 1993; Muizelaar, 1994) where six month GOS was not assessed. This imputed version of the six month GOS $(\mathrm{N}=8721)$ was used for all of the initial analyses reported in this series of papers published in this issue of the Journal of Neurotrauma.

\section{DISCUSSION}

With few exceptions, the data acquired during the last three decades in organized head injury studies has been harvested, formatted, and electronically stored by the IMPACT study group. Many thought that this would not be possible because of the wide variety of definitions, classifications, and clinical record forms that were used in data acquisition. To some degree, uniformity could not be established since many pharmaceutical companies who conducted the trials were bound to duplicate the data format with which their in-house staff was familiar. Likewise, as clinicians became more aware of new variables that made sense with regard to identifying the severity of injury or classifying outcome, new forms were generated to capture these data. An example was the Marshall CT classification, which was developed during the implementation of the TCDB. The classification was gradually introduced as a variable in subsequent studies, including HIT II, TIUS, TINT, SLIN, EBIC, SKB, and SAPHIR. Also, the formation of the brain injury consortia, in the United States (ABIC) and later in Europe (EBIC), had a major influence in trying to standardize aspects of the data collection. As a result, many of the more current variables such as cerebral perfusion pressure and neuroworsening, describing the acute course of the head injured patient were included in the more recent pharmaceutical trial data acquisition.

\section{Quality of Data within IMPACT}

The first observational study sponsored by the NIH was the TCDB (Foulkes et al., 1991; Marshall et al., 1983). In this observational study, rigorous quality control was instituted by the NIH Biometrics group and each investigator received a weekly printout of data errors that had to be rectified. The TCDB also introduced the "central reader" concept for analysis of CT pathology. As this was a funded study, adequate staff were available to ensure that the data collected were an accurate reflection of the source documents at each of the four participating institutions. The same was true for the pharmaceutical trials where clinical research organizations (CROs) provided staff to visit participating institutions and to compare clinical record forms against source documents to assure the quality of data capture. When it is estimated that approximately 20-25 million U.S. dollars was required to conduct a clinical trial in TBI, a great portion of this amount dedicated to data collection, processing, cleansing and querying, the underlying cost of the databases comprising IMPACT is considerable and approximates 220 million U.S. dollars. In summary, the data within IMPACT is of as high a standard as can realistically be achieved, as it has been subjected to intense scrutiny by groups funded to provide a high level of quality control.

\section{Problems in Development of the IMPACT Database}

The problems of merging data were extensive as there was no uniformity in data collection. The size of the data dictionary required to tabulate all of the formats attests to the many definitions utilized by each study. For example, the GOS in many studies adopted a 1-5 scale for death, vegetative state, severe disability, moderate disability and good outcome with 1 being dead. Others used the reverse, where dead was assigned the number 5. For 
this reason, a considerable effort was made in completing the data dictionary which contained the variable name, database name, variable label, variable values as defined in the clinical research forms, specific comments regarding the data and the specific form used by the trial. A major effort was required to merge the TPDS where the variables were limited to those necessary to complete the analyses set out in our original grant application to the NIH. Further data can be extracted as specified in the data dictionary should we wish to tackle other research questions.

\section{Future Plans for IMPACT}

The current IMPACT database covers the period from 1984 to 1997 . As new studies are completed, requests will be made to incorporate them into the IMPACT database. We have been granted access to the NABIS-Hypothermia study (Clifton et al., 2001) and to the Cerestat study, a phase III trial on the neuroprotective effect of Aptiganel HCL, a non-competitive antagonist of the NMDA-ion channel. These studies will be included in an expanded version of the IMPACT database. We consider it of major importance to obtain formal studies that have used the extended Glasgow Outcome Scale (GOSE) (Wilson et al., 1998) as this potentially offers an outcome measure of greater sensitivity than the current standard GOS. Plans are in place for incorporating data from the recently completed PHARMOS study of Dexanabinol where GOSE was recorded (Maas et al, 2006). Similarly, we are exploring the incorporation of the CRASH megatrial (Edwards et al., 2005) of some 10,000 patients testing the utility of steroid therapy. Although, data collection was simplified in the CRASH study, it contains valuable information on severe brain injury and will be an excellent resource for testing the relative utility of small well focused trials versus the mega-trial approach. Meanwhile, IMPACT investigators have completed univariate and multivariate prognostic analysis. This effort will subsequently lead to an expansion of preliminary work in developing a prognostic score to estimate six month outcome (Hukkelhoven et al., 2005; Maas et al., 2004). The prognostic score will then form the basis of applying the sliding dichotomy concept (Murray et al., 2005) for a more sensitive method of detecting a difference with a therapeutic intervention. It is here where IMPACT will be an essential tool in forging new approaches to clinical trial design.

\section{ACKNOWLEDGMENTS}

The IMPACT investigators wish to express their sincere gratitude to the principal investigators and stake- holders of the observational studies and RCTs for granting us permission to include their datasets for our studies. Further, the investigators are grateful to Dr. Rodrigo Labouriau (Denmark) for his help in extracting data for the Tirilazad trials. Grant support was provided by NS042691.

\section{REFERENCES}

BAILEY, I., BELL, A., GRAY, J., et al. (1991). A trial of the effect of nimodipine on outcome after head injury. Acta Neurochir. 110, 97-105.

BERETTA, I., GRANDI, E., CITERIO, G., CORMIO, M., and STOCCHETTI, N. (2003). Neuro-link, an Italian traumatic coma data bank: what did we learn from the first 1000 patients and how can we do better? Minerva Anestesiol. 69, 223-226.

BRAAKMAN, R., HABBEMA, J.D., GELPKE, G.J., and MINDERHOUD, J.M. (1980). Important factors for the prognosis of severe brain injuries; design and various results of an international study. Nederlands Tijdschrift Geneeskunde. 124, 548-555.

CITERIO, G., STOCCHETTI, N., CORMIO, M., and BERETTA, L. (2000). Neuro-Link, a computer-assisted database for head injury in intensive care. Acta Neurochir. 142, 769-776.

CLIFTON, G.L., MILLER, E.R., and CHOI, S.C., et al. (2001). Lack of effect of induction of hypothermia after acute brain injury. N. Engl. J. Med. 344, 556-563.

EDWARDS, P., ARANGO, M., and BALICA, L., et al. (2005). Final results of MRC CRASH, a randomised placebo-controlled trial of intravenous corticosteroid in adults with head injury-outcomes at 6 months. Lancet 365, 1957-1959.

EUROPEAN STUDY GROUP ON NIMODIPINE IN SEVERE BRAIN INJURY. (1994). A multicenter trial of the efficacy of nimodipine on outcome after severe head injury. J. Neurosurg. 80, 797-804.

FEARNSIDE, M.R., COOK, R.J., McDOUGALL, P., and LEWIS, W.A. (1993). The Westmead Head Injury Project. Physical and social outcomes following severe head injury. Br. J. Neurosurg. 7, 643-650.

FOULKES, A.M., EISENBERG, M.H., and JANE, A.J. (1991). The Traumatic Coma Data Bank: design, methods, and baseline characteristics. J. Neurosurg. 75, s8-s15.

HUKKELHOVEN, C.W., STEYERBERG, E.W., and HABBEMA, J.D., et al. (2005). Predicting outcome after traumatic brain injury: development and validation of a prognostic score based on admission characteristics. J. Neurotrauma 22, 1025-1039.

JENNETT, B., and BOND, M. (1975). Assessment of outcome after severe brain damage. Lancet 1, 480-484. 


\section{MARMAROU ET AL.}

MAAS, A.I.R., MARMAROU, A., MURRAY, G.D., and STEYERBERG, E.W. (2004). Clinical trials in traumatic brain injury: current problems and future solutions. Acta Neurochir. Suppl. 89, 113-118.

MAAS, A.I.R., MARMAROU, A., MURRAY, G.D., TEASDALE, G.M., and STEYERBERG, E.W. (2007). Prognosis and clinical trial design in traumatic brain injury: The IMPACT study. J. Neurotrauma 24, 232-238.

MAAS, A.I.R., MURRAY, G.D., HENNEY, H., et al. (2006b). Efficacy and safety of dexanabinol in severe traumatic brain injury: results of a phase III randomized, placebo-controlled, clinical trial. Lancet Neurol. 5, 38-45.

MARMAROU, A. (1996). Conduct of head injury trials in the United States: the American Brain Injury Consortium (ABIC). Acta Neurochir. Suppl. 66, 118-121.

MARMAROU, A., NICHOLS, J., BURGESS, J., et al. (1999). Effects of the bradykinin antagonist Bradycor (deltibant, CP1027 ) in severe traumatic brain injury: results of a multi-center, randomized, placebo-controlled trial. American Brain Injury Consortium Study Group. J. Neurotrauma 16, 431-444.

MARSHALL, L.F. (1988). The role of aggressive therapy for head injury: does it matter? Clin. Neurosurg. 34, 549-559.

MARSHALL, L.F., BECKER, D.P., and BOWERS, S.A., et al. (1983). The National Traumatic Coma Data Bank. Part 1: Design, purpose, goals, and results. J. Neurosurg. 59, 276284.

MARSHALL, L.F., MAAS, A.I., and MARSHALL, S.B., et al. (1998). A multicenter trial on the efficacy of using tirilazad mesylate in cases of head injury. J. Neurosurg. 89, 519-525.

MORRIS, G.F., BULLOCK, R., MARSHALL, S.B., MARMAROU, A., MAAS, A., and MARSHALL, L.F. (1999). Failure of the competitive $N$-methyl-D-aspartate antagonist Selfotel (CGS 19755) in the treatment of severe head injury: results of two phase III clinical trials. The Selfotel Investigators. J. Neurosurg. 91, 737-743.

MUIZELAAR, J.P. (1994). Clinical trials with Dismutec (pegorgotein; polyethylene glycol-conjugated superoxide dismutase; PEG-SOD) in the treatment of severe closed head injury. Adv. Exp. Med. Biol. 366, 389-400.

MUIZELAAR, J.P., KUPIEC, J.W., and RAPP, L.A. (1995). PEG-SOD after head injury. J. Neurosurg. 83, 942.

MUIZELAAR, J.P., MARMAROU, A., YOUNG, H.F., et al. (1993). Improving the outcome of severe head injury with the oxygen radical scavenger polyethylene glycol-conjugated superoxide dismutase: a phase II trial. J. Neurosurg. 78, 375-382.
MUIZELAAR, J.P., and MILLER, L.P. (2001). Clinical trials in head injury: United States, in: Head Trauma Basics: Preclinical and Clinical Directions. L.P. Miller and R.L. Hayes (eds), Wiley-Liss: New York, pps. 417-432.

MURRAY, G.D., BARER, D., and CHOI, S., et al. (2005). Design and analysis of phase III trials with ordered outcome scales: the concept of the sliding dichotomy. J. Neurotrauma 22, 511-517.

MURRAY, G.D., TEASDALE, G.M., and BRAAKMAN, R., et al. (1999a). The European Brain Injury Consortium survey of head injuries. Acta Neurochir. 141, 223-236.

MURRAY, L.S., TEASDALE, G.M., MURRAY, G.D., MILLER, D.J., PICKARD, J.D., and SHAW, M.D. (1999b). Head injuries in four British neurosurgical centres. Br. J. Neurosurg. 13, 564-569.

NARAYAN, R.K., MICHEL, M.E., and ANSELL, B., et al. (2002). Clinical trials in head injury. J. Neurotrauma 19, 503-557.

SCHOUTEN, J.W., FULP, C.T., and ROYO, N.C., et al. (2004). A review and rationale for the use of cellular transplantation as a therapeutic strategy for traumatic brain injury. J. Neurotrauma 21, 1501-1538.

TEASDALE, G., and JENNETT, B. (1974). Assessment of coma and impaired consciousness. A practical scale. Lancet 2, 81-84.

TEASDALE, G.M., BRAAKMAN, R., COHADON, E., et al. (1997). The European Brain Injury Consortium. Nemo solus satis sapit: nobody knows enough alone. Acta Neurochir. (Wien) 139, 797-803.

WILSON, J.T., PETTIGREW, L.E., and TEASDALE, G.M. (1998). Structured interviews for the Glasgow Outcome Scale and the extended Glasgow Outcome Scale: guidelines for their use. J. Neurotrauma 15, 573-585.

YOUNG, B., RUNGE, J.W., and WAXMAN, K.S., et al. (1996). Effects of pegorgotein on neurologic outcome of patients with severe head injury. A multicenter, randomized controlled trial. JAMA 276, 538-543.

Address reprint requests to: Anthony Marmarou, Ph.D. Virginia Commonwealth University Health Systems Department of Neurosurgery 1001 East Broad Street, Suite 235 Richmond, VA 23219

E-mail: Marmarou@hsc.vcu.edu 